\title{
El Currículum oculto en la educación universitaria: Un estudio de caso en la Universidad de Panamá
}

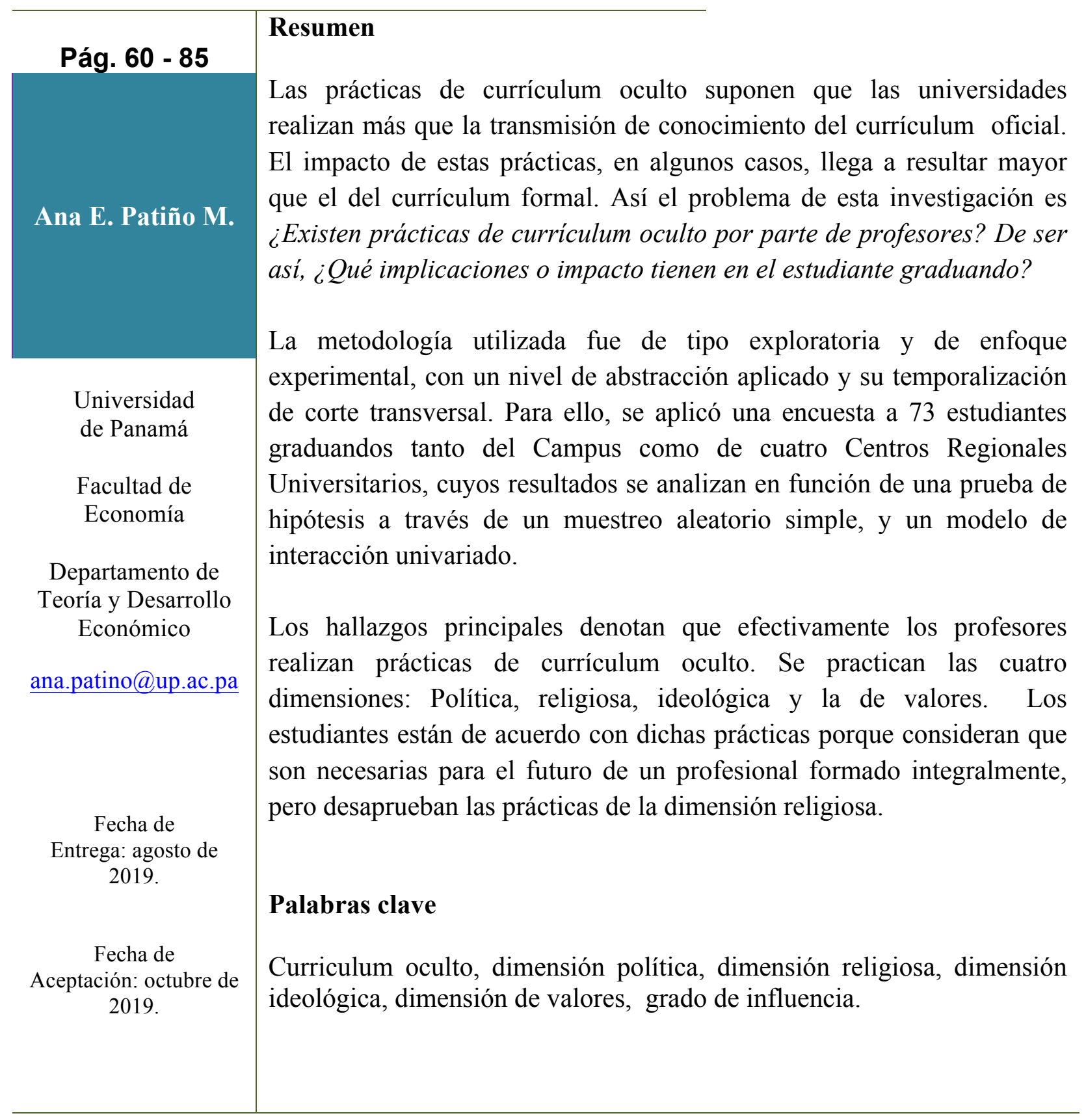




\title{
The Hidden Curriculum in Higher Education: A Case Study at the Universidad de Panama
}

\begin{abstract}
The practice of hidden curriculum implies that universities carry out more than transferring knowledge through their prescribed curricula. The impact of these practices, in some cases, happen to be greater than those included in the formal curriculum. So, the problem of this research is: Do professors turn to hidden curriculum practices in their teaching? If so, what implications or impact do such practices have on senior students?

We used an exploratory type methodology and an experimental approach, with an applied level of abstraction and its cross-sectional temporization. To achieve it, a survey was applied to 73 senior students both at the main Campus and at four Regional University Campuses, whose results are analyzed based on a hypothesis test through a simple random sampling, and a univariate interaction model.
\end{abstract}

The main findings evidence that in fact teachers carry out hidden curriculum practices in four dimensions: political, religious, ideological and values. Students agree with these practices because they consider them as necessary for the future of a fully trained professional, but they disagree with the practices in the religious dimension.

\section{Keywords}

Hidden curriculum, political dimension, religious dimension, ideological dimension, dimension of values and degree of influence.

REVISTA ANUAL ACCIÓN Y REFLEXIÓN EDUCATIVA, N 45

enero, 2020

SSN L 2664-3775 
El Currículum oculto en la educación universitaria:

Un estudio de caso en la Universidad de Panamá

\section{Introducción}

El propósito de este artículo consiste en presentar los resultados de una investigación, que buscaba comprobar si los profesores universitarios realizan prácticas de curriculum oculto y posteriormente, analizar el impacto de dichas prácticas en sus estudiantes graduandos. Estas prácticas expresan la idea de que subyacen impactos sociales, políticos, ideológicos y culturales en las actividades educativas y en muchas ocasiones su impacto llega a resultar mayor que el del currículum oficial. Para esta investigación se seleccionó una unidad académica del campus central de la Universidad de Panamá y cuatro centros regionales universitarios.

La Universidad de Panamá es una institución que se caracteriza por la realización de debates, foros y congresos orientados a resolver distintos problemas a nivel nacional y muchos de sus profesores(as) han jugado importantes roles en escenarios de la vida política y administrativa a nivel privado y público. El artículo presenta un abordaje teórico y conceptual, posteriormente se fundamenta la metodología del diseño de la investigación y por último se presentan los resultados y principales hallazgos.

\section{Abordaje teórico - conceptual}

El abordaje o marco referencial epistemológico facilita el establecimiento de hipótesis y por ende en la producción del nuevo conocimiento. En este sentido hacemos referencia al concepto de currículum oculto y los autores Henry Giroux y Jurjo Torres Santomé. En cuanto a la conceptualización, el curriculum ha sido presentado de diferentes maneras; es frecuente encontrarlo como un proceso, como un producto, o como ambos. Sin embargo, los teóricos del currículum han descrito que, de manera paralela o alternativa al currículum formal, existe otro currículum denominado "currículo oculto", término acuñado en 1968 por el educador norteamericano Philip W. Jackson; quien al respecto indica:

REVISTA ANUAL ACCIÓN Y REFLEXIÓN EDUCATIVA, N 45

enero, 2020

ISSN L 2664-3775 
...la multitud, el elogio y el poder que se combinan para dar un sabor específico a la vida en el aula forman colectivamente un currículum oculto que cada alumno (y cada profesor) debe dominar para desenvolverse satisfactoriamente en la escuela. Las demandas creadas por estos rasgos de la vida en el aula pueden contrastarse con las demandas académicas (el currículum “oficial”, por así decirlo) a las que los educadores tradicionalmente han prestado mayor atención. Como cabía esperar, los dos currículos se relacionan entre sí de diversos e importantes modos (Jackson, 2001, p.73).

Por su parte, John Eggleston (1980), afirma que el currículo oculto no constituye nada nuevo, ya que se advierte su presencia en la reforma del siglo XIX; sostiene que tanto alumnos como profesores deben aprender este currículo que califica de esencial, si es que quieren sobrevivir en el salón de clase y lograr siquiera un principio de participación en el currículo oficial. En esta misma línea, José D. Huerta Peña, (1988) plantea que, entendemos al currículo oculto como un proceso de enseñanza aprendizaje, paralelo e implícito al currículo formal que se expresa de manera cotidiana, consciente o inconscientemente en el ámbito escolar a través de los valores, mensajes, actitudes e intereses de quienes conviven el proceso educativo formal, legitimando con él la cultura y el poder del sistema dominante.

María José López Da Silva (1996) plantea que hoy en día podemos encontrar ocupando los banquillos escolares a los hijos de los trabajadores, a los de la clase media y a los de la burguesa. Esto puede provocar la ilusión de que todo el mundo tiene las mismas oportunidades de educación. Pero vamos a verificar que esto no es realmente cierto, se trata tan solo de la implementación de mecanismos ideológicos que se van a producir al interior de la escuela, cuyo objetivo es perpetuar las desigualdades sociales, las diferencias de clase existentes en la sociedad; uno de estos mecanismos es el llamado currículo oculto.

REVISTA ANUAL ACCIÓN Y REFLEXIÓN EDUCATIVA, N 45

enero, 2020

SSN L 2664-3775 
El Currículum oculto en la educación universitaria:

Un estudio de caso en la Universidad de Panamá

Una vez planteadas las anteriores conceptualizaciones, podemos elaborar un constructo propio de currículo oculto: Es el currículo paralelo, currículo vivido, currículo real; asumiendo que el currículo oculto son todos los elementos que, sin estar contemplados en el currículum formal de la institución, se inculca a través de la práctica docente cotidiana en la educación superior, que incluso, puede llegar a tener mayor significación que los aprendizajes explícitos en el currículo oficial.

Como referencia teórica, presentamos el planteamiento de Jurjo Torres Santomé que, junto al enfoque de Henry Giroux, se posicionan como el basamento teórico de la investigación. El enfoque crítico del pedagogo español Torres, activista político y profesional crítico, se evidencia en 1991, cuando publica una investigación sobre currículum oculto. El sostiene que en toda sociedad las clases sociales y/o grupos que detentan el poder tratan de imponer y legitimar su dominio y de organizar su reproducción mediante ambos tipos de discursos en los diferentes escenarios donde se desenvuelve la actividad humana, contando para ello con la ayuda imprescindible del Estado. Uno de esos escenarios es la institución escolar o educativa, institución que ha denominado como Aparato Ideológico de Estado, afirmando que desempeña, en todos sus aspectos, la función dominante, adicionales a los restantes aparatos ideológicos de Estado (el religioso, jurídico, político, sindical, de la información y cultural).

Puntualiza, que hoy en día, el centro educativo prepara para la vida, pero una vida de automatización, en la que el valor radica en el consumo y, por ende, reafirma la separación de clase y viene la pobreza. Los gobiernos empresariales dan pie a que este tipo de currículo se aplique y además del olvido de la enseñanza pública para dar entrada a la privada-religiosa, no laica. Finalmente, señala que las instituciones educativas son las principales encargadas de la reproducción social, de correspondencia y cultural del sistema capitalista, la cual desempeña tan bien su función de preservar las clases sociales.

En segunda instancia, presentamos a Henry Giroux, que publica el libro Los profesores como intelectuales: Hacia una pedagogía crítica del aprendizaje (1997), que sirve de base o 
fundamento teórico para definir una tríada de enfoques-paradigmas teóricos en la pedagogía y hace un abordaje que sustenta la importancia de las prácticas del currículum oculto, especialmente en el campo de las ciencias económicas. En la visión de Giroux, la institución educativa no es, como entienden otros enfoques, un ámbito autónomo, sino que se halla engarzado en la mayor complejidad de la sociedad y economía que la han producido; así cualquier análisis que hagamos de la institución educativa se explica desde una perspectiva teórica que se pronuncia también, de un modo u otro, acerca de la sociedad, la verdad y la realidad en general.

Se trata de la filosofía que subyace a cualquier producción humana, también la acción y producción humana (economía), que lejos de cómo lo ve el enfoque estatista que emana del paradigma neoliberal. Giroux exalta la pedagogía crítica, como teoría que propone que los estudiantes, a través de la práctica, alcanzan una conciencia crítica dentro de su sociedad; una pedagogía liberadora como un vehículo para la construcción de la razón crítica que les permita a los ciudadanos romper con lo predefinido, es decir, que sean capaces de tomar una posición crítica frente a la sociedad y su dominación existente a causa del seguimiento de las pedagogías tradicionales.

Posteriormente publica: Teoría y Resistencia en Educación: Una pedagogía para la oposición (Giroux, 1997), donde critica el discurso convencional de la alfabetización, ya que éste la define en términos mecánicos y/o funcionales; la concibe como la simple adquisición de ciertas habilidades relacionadas con el lenguaje escrito y la sumerge a la lógica y las necesidades del capital, midiendo su valor según la demanda de dichas habilidades de lectura y escritura necesarias para el crecimiento del sector trabajo. Al respecto, Giroux (1992) señala que:

Tanto la alfabetización como la escolarización misma son parte de un fenómeno político, $\mathrm{y}$ en parte representan un terreno epistemológico asediado en el que diferentes grupos sociales luchan

REVISTA ANUAL ACCIÓN Y REFLEXIÓN EDUCATIVA, N 45 enero, 2020 
El Currículum oculto en la educación universitaria:

Un estudio de caso en la Universidad de Panamá

por la forma en que ha de ser expresada, reproducida y resistida, consecuentemente, la alfabetización en términos convencionales ha caído bajo el peso de la ideología operacional que da forma y legitima a la lógica de la sociedad dominante (p. 260).

Para Giroux, el currículum oculto se encuentra constituido por las normas, valores, y creencias no afirmadas explícitamente que se transmiten a los estudiantes a través de las estructuras subyacentes tanto del contenido formal como de las relaciones de la vida escolar y del aula. En otras palabras, la escuela es mucho más que los contenidos formales de cada asignatura y las herramientas didácticas empleadas por los maestros y profesores para transmitir dichos contenidos.

\section{Abordaje metodológico}

Esta investigación es exploratoria y de enfoque experimental, tiene un nivel de abstracción aplicado, sigue un procedimiento de comprobación experimental para rechazar o aceptar la hipótesis de que las prácticas de currículum oculto de los profesores impactan positivamente en los estudiantes graduandos. Implicó dos componentes para el análisis; el primero es un muestreo aleatorio simple y el segundo, una experimentación con las dos variables de la investigación, las cuales serán analizadas a través del método estadístico Análisis de Varianzas (Anova). Las fuentes primarias, provienen de las encuestas validadas aplicadas a estudiantes de la unidad académica seleccionada, distribuidos en el Campus Central y los respectivos Centros Regionales Universitarios. Se decidió encuestar a estudiantes graduandos, ya que estos han tenido la oportunidad a lo largo de su carrera de conocer y tratar a muchos profesores; adicional a ello poseen mayor madurez, conocimiento y experiencia en el llenado del cuestionario.

REVISTA ANUAL ACCIÓN Y REFLEXIÓN EDUCATIVA, N 45

enero, 2020

ISSN L 2664-3775 


\section{Dimensiones de curriculum oculto analizadas}

Las cuatro dimensiones del currículum oculto que se consideraron en esta investigación son la dimensión religiosa, la dimensión política, la dimensión ideológica y la ética y valores, que para los fines de estudio se delimitó como sigue:

- Dimensión Religiosa: para evidenciar su práctica se deben desarrollar algunas de las siguientes prácticas: Defensa o critica de la laicidad, del cristianismo, del ateísmo y de otras religiones.

- Dimensión Política: para evidenciar la práctica se debe desarrollar algunas de las siguientes prácticas: Defensa o critica a la política gubernamental. defensa o critica de la política partidista, defensa o critica de la política ambientalista y defensa o critica a la política con enfoque de género.

- Dimensión Ideológica/filosófica: para evidenciar su práctica se deben desarrollar algunas de las siguientes prácticas: Defensa o crítica del comunismo y socialismo, defensa o critica del capitalismo defensa o critica del neoliberalismo y defensa o critica el eclecticismo.

- Dimensión de Ética y Valores: para evidenciar esta práctica se deben desarrollar algunas de las siguientes prácticas: Discusión de valores fundamentales, de valores humanos y personales, de valores morales y de valores cívicos y culturales.

\section{Diseño de la investigación}

La investigación tiene dos componentes de análisis, el primero es una prueba de hipótesis a través de un muestreo aleatorio simple, y el segundo, es un modelo de interacción univariado. El muestreo aleatorio simple se utilizó para demostrar la existencia de prácticas de currículum oculto por parte de los profesores, basados en el planteamiento de las siguientes hipótesis:

REVISTA ANUAL ACCIÓN Y REFLEXIÓN EDUCATIVA, N 45

enero, 2020

SSN L 2664-3775 
El Currículum oculto en la educación universitaria:

Un estudio de caso en la Universidad de Panamá

- $\quad \mathbf{H}_{\mathbf{0}}: \boldsymbol{\mu} \leq \mathbf{5 0 \%}$ Hipótesis Nula: "Los profesores no desarrollan prácticas de currículum oculto". El 50\% o menos de los encuestados afirmaron que sus profesores realizan prácticas de Currículum Oculto.

- $\mathbf{H}_{1}: \boldsymbol{\mu}>$ 50\%Hipótesis Alternativa: "Los profesores desarrollan prácticas de currículum oculto". Más del 50\% de los encuestados afirmaron que sus profesores realizan prácticas de Currículum Oculto.

El cálculo de la muestra resultó de 73 estudiantes, considerando una población finita de 225 , el valor de la distribución de Gauss, $Z \alpha=0.05=1.96$, el grado de confianza es del $95 \%$ y su error máximo permitido (e) es del 5\%. Se prevé que el 95\% de los estudiantes se ven impactados de alguna forma por las prácticas de currículum oculto, por ello la proporción (p) es de 0.95.

El modelo de interacción univariado se desarrolló a través del método estadístico de Análisis de la Varianza (ANOVA) unifactorial, que estudia simultáneamente los efectos de variación e interacción entre las variables en análisis no son nulas ANOVA es una técnica estadística que señala si dos variables (independiente y dependiente) están relacionadas.

Es decir, compara las medias de la variable dependiente entre los grupos o categorías de la variable independiente.

La Hipótesis del Modelo ANOVA supone que las interacciones entre las variables factores no son nulas, la interacción de las variables causa efectos una sobre la otra, por lo tanto, se tiene la siguiente hipótesis para demostrar la a probabilidad resultante debe ser menor de 0.05 para aceptar Ho y la probabilidad debe ser mayor de 0.05, para rechazar Ho.

Hipótesis: Efectos del tratamiento

- $\mathbf{H}_{0}: \mathbf{H}_{0}: \tau_{1}=\tau_{2}=\ldots \tau_{\mathrm{a}}=\mathbf{0}$ No existe diferencia significativa entre la media de los tratamientos, es decir que son iguales.

REVISTA ANUAL ACCIÓN Y REFLEXIÓN EDUCATIVA, N 45

enero, 2020

ISSN L 2664-3775 
- $\mathbf{H}_{1}: \mathbf{H}_{1}$ : al menos una $\tau_{\mathbf{i}} \neq \mathbf{0}$ Existe diferencia significativa entre la media de los tratamientos, es decir que son diferentes.

\section{Análisis de los resultados del muestreo}

a. Aspectos generales de los encuestados(as): Del total de los encuestados(as), veinte pertenecen a Campus Central, quince del Centro Regional de Los Santos (CRULS), once del Centro Regional de Azuero (CRUA), catorce del Centro Regional de Coclé (CRU Coclé) y trece del Centro Regional de San Miguelito (CRUSAM). Por motivos presupuestarios, no se pudo llegar a encuestar en los demás Centros Regionales Universitarios ni en las Extensiones Universitarias. Estos datos se presentan en la Figura 1.

De los encuestados(as) el 68\% (50 estudiantes) son mujeres y el restante 32\%, hombres. Esta realidad se corresponde con los datos estadísticos de egreso de la Universidad de Panamá, los cuales plantean que siete de cada diez egresados universitarios son mujeres. Un factor importante que incide en las respuestas es la edad de los encuestados(as), dado a que sus respuestas están condicionadas por la experiencia y madurez de cada uno de los estudiantes. En este sentido, se puede apreciar que la mayor parte de los encuestados(as) el 60\% (44 estudiantes) tiene entre 26 y 28 años de edad, en tanto que en menor grado se encuestaron estudiantes mayores de 30 años de edad. 


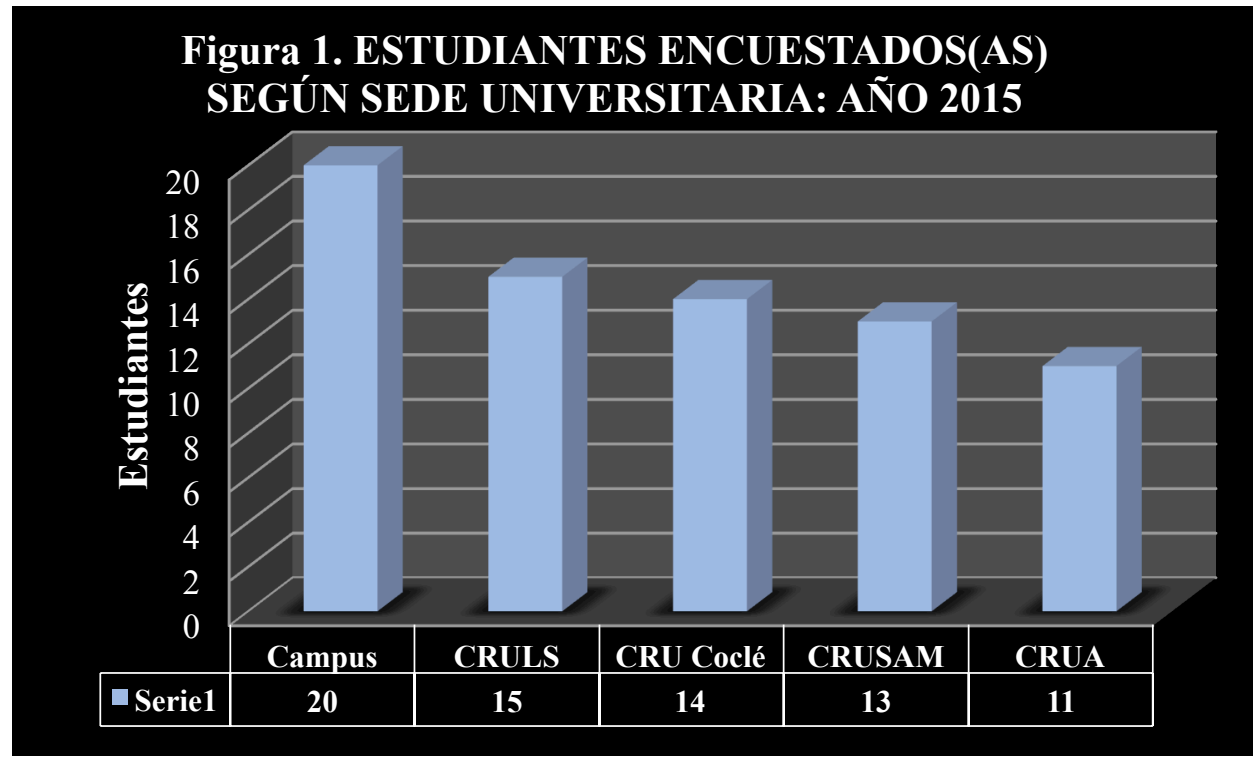

Fuente: La autora en base a datos provenientes de las encuestas aplicadas.

Dado, que las preguntas acerca de las dimensiones de currículum oculto son de índole religiosa, política, ideológica y de valores; se intentó conocer el perfil de los encuestados(as) en cuanto a estas dimensiones. Así tenemos que, en cuanto a la religión, el 100\% de los encuestados profesa alguna fe religiosa, extrañamente, ninguno confesó ser ateo. El 67\% manifestó ser católico y el restante 33\%, evangélicos. Estos resultados coinciden con el promedio nacional ya que en Panamá la religión católica es mayoritaria.

Un resultado distinto se obtuvo sobre la adscripción política de los encuestados(as), en este sentido un significativo 30\% manifestó no estar inscrito en ningún partido, a pesar de que la mayoría de los encuestados(as), ha pasado un promedio de 2 torneos electorales desde su mayoría de edad. A pesar de poseer el dinamismo de la juventud y el espíritu de la participación política, solo el $25 \%$ de los encuestados participan en algún club cívico y el restante $75 \%$ no se encuentra activo en ninguna asociación de interés cívico, cultural o deportivo. Resultados inversos se obtuvieron en la participación política e ideológica, ya que el 68\% indicó tener una ideología definida, por lo que se deduce que se debe a la orientación político-ideológica que recibe en su colectivo político. 
b. Demostración de las prácticas de curriculum oculto: Los resultados de la encuesta arrojan que el $84.93 \%$ de los encuestados(as), 62 estudiantes, afirma que sus profesores realizan prácticas de curriculum oculto; un $10.95 \%$ manifestó que no se practican y apenas un $4.1 \%$ no supo identificar con claridad si las prácticas se dan o no por parte de los profesores. Estos datos se presentan en la Figura 2.

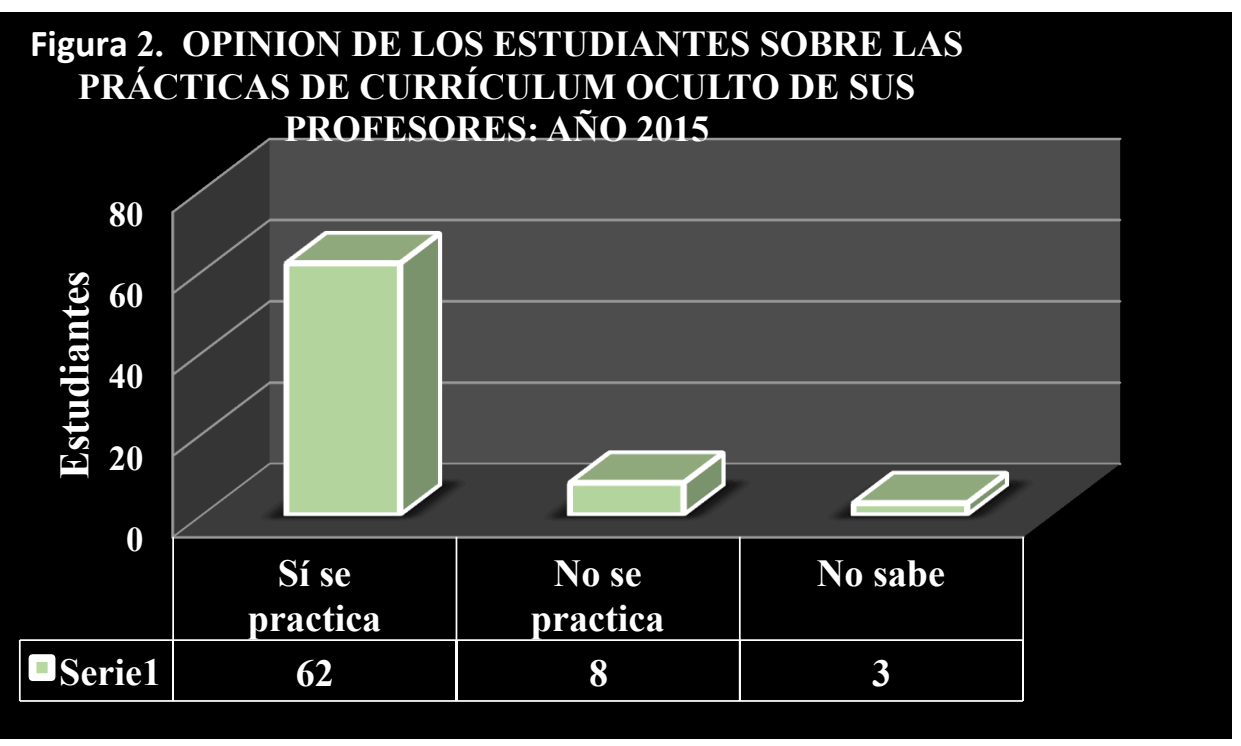

Fuente: La autora en base a datos provenientes de las encuestas aplicadas.

De los 62 estudiantes que afirman que sus profesores desarrollan prácticas de Curriculum Oculto, diecinueve estudian en el Campus Central, trece en el CRU Coclé, doce en CRULS, once en el CRUA y siete en el CRUSAM. Esta distribución se presenta en la Figura 3. 
El Currículum oculto en la educación universitaria:

Un estudio de caso en la Universidad de Panamá

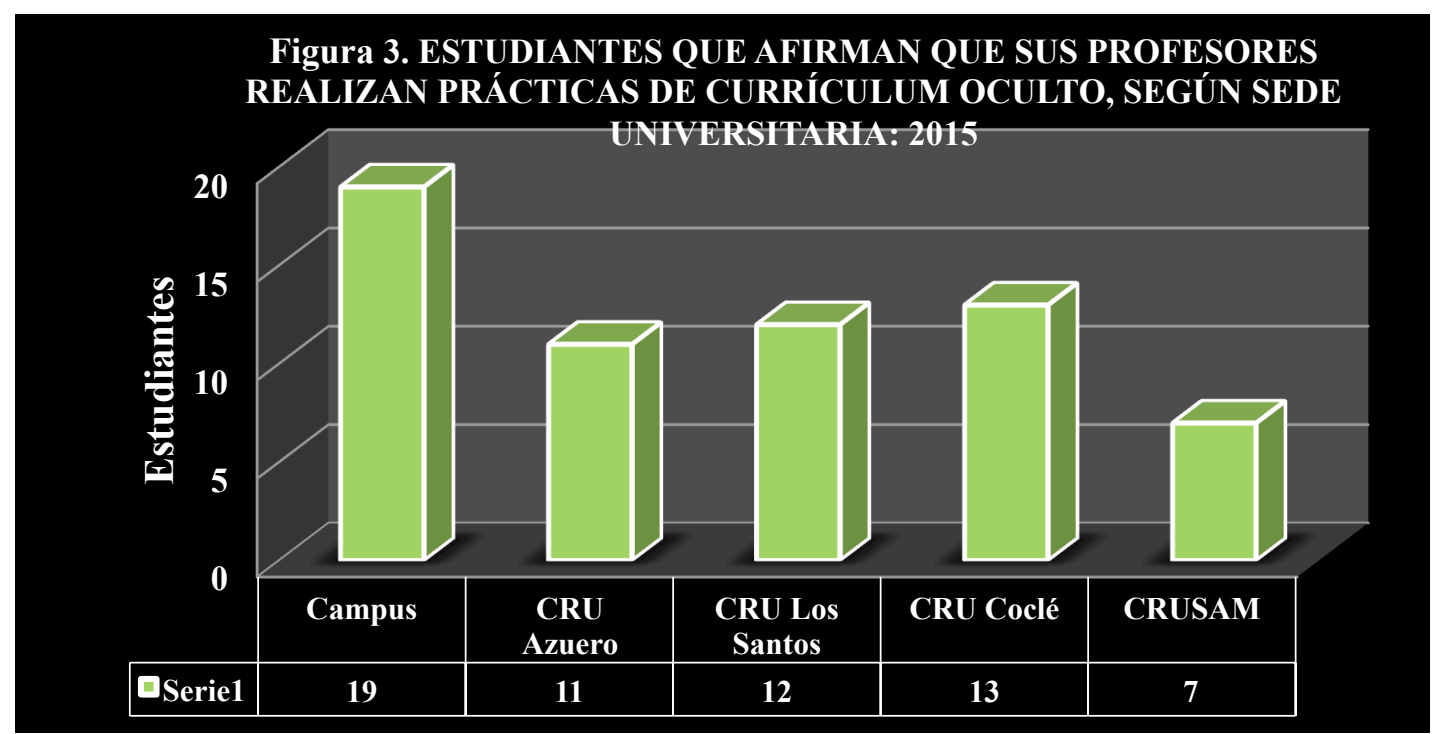

Fuente: La autora en base a datos provenientes de las encuestas aplicadas.

\section{c. Prueba de Hipótesis del muestreo aleatorio simple}

Esta prueba implica la identificación del valor estadístico de prueba $Z$, para ello se utilizó un nivel de significación de 5\% $(\alpha)$ para investigaciones de tipo social, lo cual arrojó un resultado de $\mathrm{Z}=8.35$. Con una confiabilidad del $95 \%$ se rechaza la Hipótesis Nula y se confirma la Hipótesis alternativa de que más del $50 \%$ de los profesores realizan prácticas de Currículum Oculto, como se aprecia en la Figura 4.

Figura 4. DISTRIBUCIÓN NORMAL CON LAS ÁREAS DE ACEPTACIÓN Y RECHAZO DE Ho

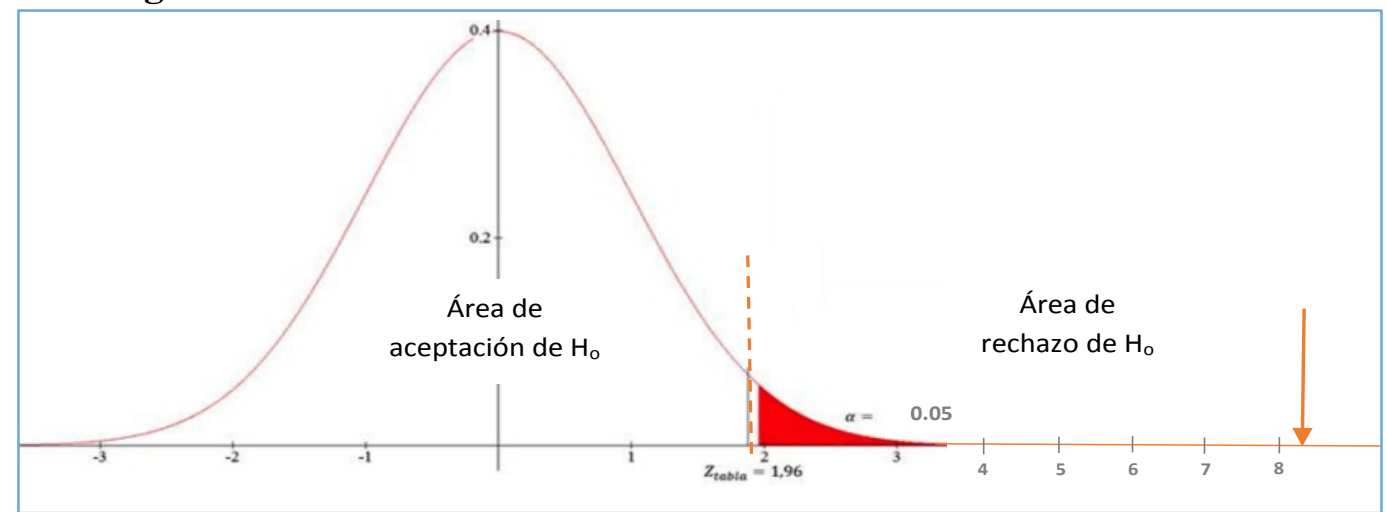

Fuente: Elaboración propia en base a las pruebas estadísticas.

REVISTA ANUAL ACCIÓN Y REFLEXIÓN EDUCATIVA, N 45

enero, 2020

ISSN L 2664-3775 


\section{Análisis de los resultados de la ANOVA}

El análisis de estadístico ANOVA, indica que los valores totales obtenidos, reflejan que los 73 estudiantes encuestados otorgaron calificaciones altas (4,571 puntos) en la frecuencia de "Siempre" a las prácticas de Currículum Oculto y calificaciones menores (2,316 puntos) en la frecuencia de "Nunca" de dichas prácticas. Ello nos lleva a concluir que las prácticas de Curriculum Oculto por parte de los profesores son frecuentes. Estos datos se presentan en la Tabla 1.

\section{Tabla 1}

VALORES TOTALES OBTENIDOS PARA LAS PRÁCTICAS DE CURRÍCULUM OCULTO, SEGÚN DIMENSIÓN: AÑOS 2015

\begin{tabular}{llllll}
\hline \multirow{2}{*}{ Dimensión } & \multicolumn{2}{l}{ Frecuencia } & & \\
& Nunca & Casi Nunca & $\begin{array}{l}\text { Algunas } \\
\text { veces }\end{array}$ & $\begin{array}{l}\text { Mayoría de } \\
\text { veces }\end{array}$ & Siempre \\
\hline Religiosa & 532 & 581 & 968 & 1,097 & 1,189 \\
Política & 524 & 630 & 948 & 1,113 & 1,175 \\
Ideológica/filosófica & 517 & 594 & 982 & 1,100 & 1,198 \\
Valores Éticos & 743 & 759 & 1,020 & 843 & 1,009 \\
Total & $\mathbf{2 , 3 1 6}$ & $\mathbf{2 , 5 6 4}$ & $\mathbf{3 , 9 1 8}$ & $\mathbf{4 , 1 5 3}$ & $\mathbf{4 , 5 7 1}$ \\
Media & 579 & 641 & 979.5 & $1,038.25$ & $1,142.75$ \\
\hline
\end{tabular}

Fuente: La autora en base a los datos provenientes de la encuesta aplicada.

De la Tabla 1, podemos concluir que las dimensiones Ideológica (1,198 puntos) y Religiosa (1,189 puntos) son las mayormente practicadas por los profesores y la Dimensión de Valores Éticos (1,009 puntos) es la menos practicada. En cuanto al grado de aceptación de las prácticas de currículum oculto, los resultados indican que los estudiantes están "De acuerdo" y "Muy de acuerdo" con las prácticas, ya que en estos grados de aceptación se observan los puntajes más altos, por un total de 1,924 puntos. Los puntajes más bajos fueron otorgados a "Muy en desacuerdo". Estos datos se desagregan en la Tabla N², por otra parte, no solo es interesante conocer si los estudiantes aceptan o no las prácticas de Curriculum Oculto, sino también específicamente, el grado de aceptación de dichas prácticas.

REVISTA ANUAL ACCIÓN Y REFLEXIÓN EDUCATIVA, N 45

enero, 2020

SSN L 2664-3775 
El Currículum oculto en la educación universitaria:

Un estudio de caso en la Universidad de Panamá

Los encuestados manifestaron estar "Muy en desacuerdo" y "En desacuerdo" con las prácticas de Currículum Oculto en la Dimensión Religiosa, es un tema que consideran no debe ser tratado en las aulas. Caso contrario ocurre con la Dimensión de Valores Éticos, con la cual están "De acuerdo" y "Muy de acuerdo" que se practique por parte de los profesores. Las dimensiones de Política e Ideología obtuvieron valores cercanos a la Dimensión de Valores Éticos, por lo cual se concluye que los estudiantes no rechazan estas dos (2) prácticas por parte de sus profesores, como se manifiesta en la Tabla 2.

Tabla 2

VALORES TOTALES OBTENIDOS PARA EL GRADO DE ACEPTACIÓN DE LAS PRÁCTICAS DE CURRÍCULUM OCULTO: AÑO 2015.

\begin{tabular}{|c|c|c|c|c|c|}
\hline \multirow[b]{2}{*}{ Dimensión } & \multicolumn{4}{|c|}{ Grado de Aceptación } & \multirow[b]{2}{*}{ 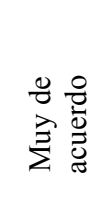 } \\
\hline & 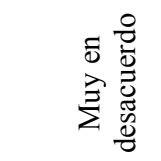 & 四 & 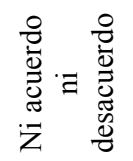 & $\begin{array}{l}8 \\
\stackrel{0}{0} \\
\overline{0} \\
\tilde{0} \\
0 \\
0\end{array}$ & \\
\hline Religiosa & 238 & 223 & 270 & 172 & 188 \\
\hline Política & 171 & 191 & 260 & 204 & 274 \\
\hline Ideológica & 151 & 154 & 241 & 266 & 281 \\
\hline Valores éticos & 148 & 161 & 247 & 250 & 289 \\
\hline Total & 708 & 729 & 1018 & 892 & 1032 \\
\hline Media & 177 & 182.25 & 254.5 & 223 & 258 \\
\hline
\end{tabular}

Fuente: La autora en base a los datos provenientes de la encuesta aplicada.

Para medir el impacto de las prácticas de currículum oculto, se preguntó a los estudiantes específicamente el tipo de influencia que consideraban ejercían estas prácticas sobre su persona. Los estudiantes respondieron estar "Muy de acuerdo" en que las prácticas cambian su forma de pensar y actuar, en que las mismas son muy necesarias para el profesional y que afectan positivamente su formación profesional. Rechazaron que las prácticas afectaran negativamente la formación profesional, ya que para este reglón se obtuvieron apenas 137 puntos. Ver Tabla 3.

REVISTA ANUAL ACCIÓN Y REFLEXIÓN EDUCATIVA, N 45

enero, 2020

ISSN L 2664-3775 


\section{Tabla 3}

VALORES TOTALES OBTENIDOS DEL GRADO DE INFLUENCIA DE LAS PRÁCTICAS DEL CURRÍCULUM OCULTO EN LA UNIDAD ACADÉMICA: AÑO 2015.

\begin{tabular}{|c|c|c|c|c|c|}
\hline \multirow[b]{2}{*}{ Tipo de Influencia } & \multicolumn{4}{|c|}{ Grado de Influencia } & \multirow[b]{2}{*}{ 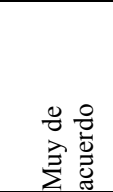 } \\
\hline & 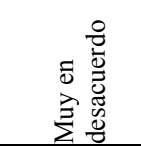 & 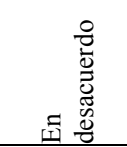 & 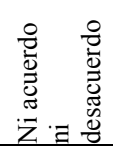 & 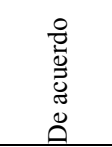 & \\
\hline Cambia su forma de pensar y actuar & 95 & 124 & 250 & 292 & 341 \\
\hline Afecta positivamente la formación profesional & 111 & 144 & 250 & 282 & 311 \\
\hline Afecta negativamente la formación profesional & 275 & 271 & 267 & 139 & 137 \\
\hline Son necesarias para el profesional & 112 & 148 & 245 & 277 & 316 \\
\hline Total & 593 & 687 & 1012 & 990 & 1105 \\
\hline Media & 148.25 & 171.75 & 253 & 247.5 & 276.25 \\
\hline
\end{tabular}

Fuente: La autora en base a los datos provenientes de la encuesta aplicada.

\subsection{Prueba de Hipótesis del análisis de varianza}

La ANOVA no requiere de la muestra total de la encuesta sino un número de réplicas (n) de la prueba con la suficiente potencia deseada. El cálculo de estas réplicas del experimento y el poder de determinada prueba $\mathbf{n}$ depende de cuatro cantidades, que son: Los grados de libertad, el nivel de significación del contraste $(\alpha=0.05)$ y la cantidad que mide el grado de $<<$ falsedad $>>$ de la hipótesis nula $(\Phi)$. Una vez estimados estos valores se determina que se requieren al menos $n=5$ réplicas para tener una prueba con la potencia deseada del $95 \%$ de confianza, de acuerdo con la Tabla 4.

\section{Tabla 4}

ESTIMACIÓN DEL PODER DE LA PRUEBA Y TAMAÑO DE LAS RÉPLICAS NECESARIAS PARA EL MODELO

\begin{tabular}{lllllll}
\hline $\mathbf{n}$ & $\Phi^{2}$ & $\Phi$ & $a-1$ & $a b(n-1)$ & $\beta$ & $\operatorname{Poder}(1-\beta)$ \\
\hline 3 & 2.76 & 1.66 & 3 & 40 & 0.35 & 0.650 \\
4 & 3.68 & 1.92 & 3 & 60 & 0.08 & 0.925 \\
$\mathbf{5}$ & $\mathbf{4 . 6 1}$ & $\mathbf{2 . 1 5}$ & $\mathbf{3}$ & $\mathbf{8 0}$ & $\mathbf{0 . 0 3}$ & $\mathbf{0 . 9 7 5}$ \\
6 & 5.53 & 2.35 & 3 & 100 & 0.01 & 0.990 \\
\hline
\end{tabular}

Fuente: Estimaciones de la autora en base a los datos provenientes de la encuesta aplicada. 
El Currículum oculto en la educación universitaria:

Un estudio de caso en la Universidad de Panamá

\subsection{Las Medias Marginales}

Para medir y evidenciar la práctica de estas dimensiones del curriculum oculto, con el apoyo del SPSS, se muestran los factores intersujetos y el correspondiente conteo de observaciones para realizar la prueba que permita tener una vista gráfica de la comparación de las medias y las medias marginales, que plantean su interacción y frecuencias con los resultados de las comparaciones múltiples. Los gráficos de las medias marginales son el valor medio de los resultados de una serie de valores que ocurren cuando se adiciona una unidad de otra variable que la afecta. Esos valores marginales se suman y dividen entre la cantidad de valores que se obtuvieron, y a eso se le llama Media de los valores marginales.

\section{Prácticas de currículum en la Dimensión Religiosa}

A pesar de que la Constitución Panameña reconoce que Panamá es un país en el cual se profesa de forma mayoritaria la religión católica; este carácter oficial otorga a la religión católica una fuerte presencia en la actividad nacional. La enseñanza del catolicismo está presente en algunas escuelas y colegios; sin embargo, las prácticas religiosas están entretejidas de herencias culturales diversas y un sincretismo de cultos, creencias africanas, animismo indígena y otras creencias que tiñen de contrastes a Panamá. En este sentido, expresamente la Constitución señala:

Es libre la profesión de todas las religiones, así como el ejercicio de todos los cultos, sin otra limitación que el respeto a la moral cristiana y el orden público. Se reconoce que la religión católica es la de la mayoría de los panameños (Constitución Política, 2004 Artículo 35).

REVISTA ANUAL ACCIÓN Y REFLEXIÓN EDUCATIVA, N 45

enero, 2020

ISSN L 2664-3775 
A esta realidad no escapa la Universidad de Panamá, por ello se observan dos tipos de resultados, por un lado, las gráficas de los tipos de prácticas de currículum oculto de la Dimensión Religiosa plantean una fuerte interacción y por el otro, las frecuencias de las prácticas no se cruzan, por tanto, no existe interacción que sea significativa, lo cual se corresponde con los resultados de las comparaciones múltiples. En el caso de las gráficas de las medias marginales podemos ver, que se confirma los valores más altos para la frecuencia de "Siempre" y la "Mayoría de las veces" y los valores más bajos para "Nunca” y "Casi nunca". Para el tipo de práctica, se evidencia que las medias marginales son muy cercanas, sin embargo, son más altas para las prácticas de "Defensa o crítica del ateísmo" y ligeramente más bajas para las prácticas de "Defensa o crítica de otras religiones". Ver Figura 5.

\section{Figura 5. Medias marginales de la Dimensión Religiosa}

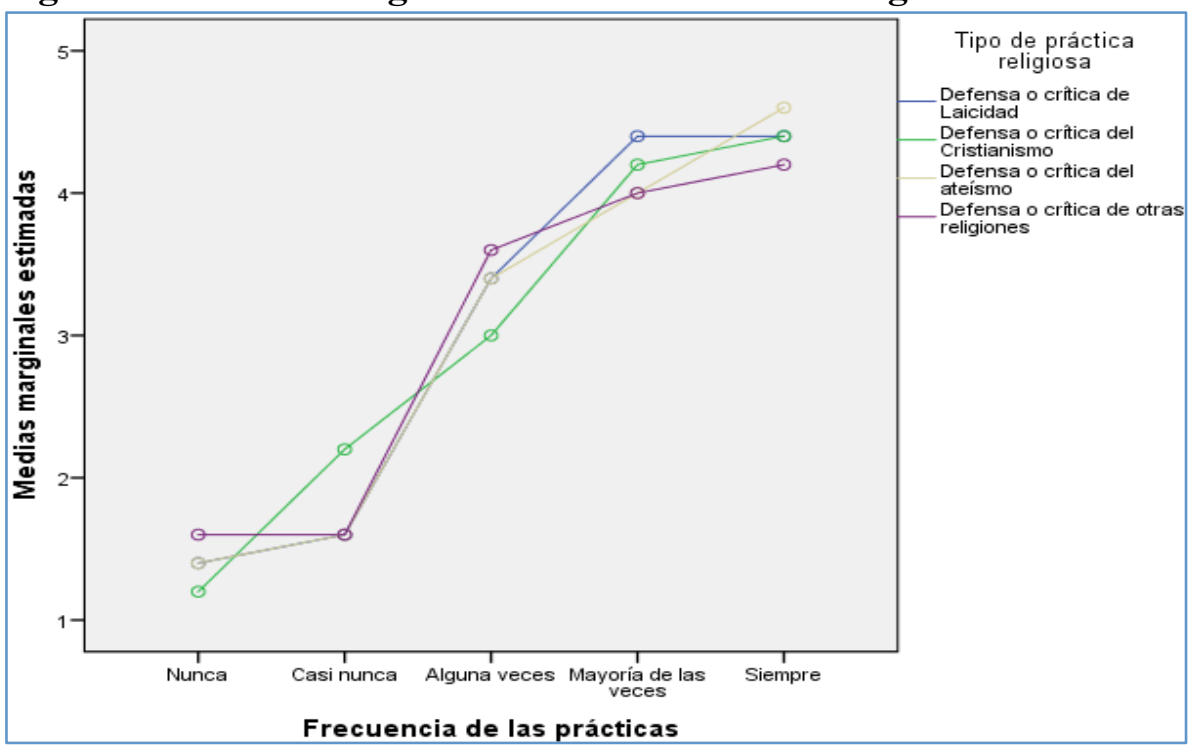

Fuente: Elaboración propia en base a las pruebas estadísticas en SPSS.

\section{Prácticas de currículum oculto en la Dimensión Política}

En términos generales, la política tiene entre otros propósitos, hacer legibles las experiencias sociales de los individuos y grupos, y sistematiza con sentido nuestros intereses individuales, subordinando el logro particular al beneficio general de la sociedad. Así, los políticos son 
El Currículum oculto en la educación universitaria:

Un estudio de caso en la Universidad de Panamá

mediadores en la formulación de estrategias que solucionan los problemas socioeconómicos que enfrenta la sociedad. Sin embargo, han sido pocos los logros en este sentido, ya que a diario se ven explosiones sociales en demanda de soluciones. Por lo anterior, es inevitable hablar de estos temas en distintos colectivos y espacios de interacción social. Uno de estos son los espacios educativos, sobre todo a nivel superior, máxime si se trata de la Universidad de Panamá, muy vinculada al acontecer socioeconómico nacional.

El análisis de la Dimensión Política presenta los gráficos de medias, donde se observan dos tipos de resultados, por un lado, las gráficas de los tipos de prácticas de currículum oculto de la Dimensión Política que plantean una fuerte interacción. En tanto, que las frecuencias de las prácticas se cruzan parcialmente, existe interacción significativa en ambos casos. Se confirma los valores más altos para la frecuencia de "Siempre" y la "Mayoría de las veces" y los valores más bajos para "Nunca" y "Casi nunca". Para el tipo de práctica, se evidencia que las medias marginales son muy cercanas, sin embargo, son más altas para las prácticas de "Defensa o crítica de la política partidista" y más bajas para la "Defensa o crítica de la política feminista".

\section{Figura 6. Medias Marginales de la Dimensión Política}

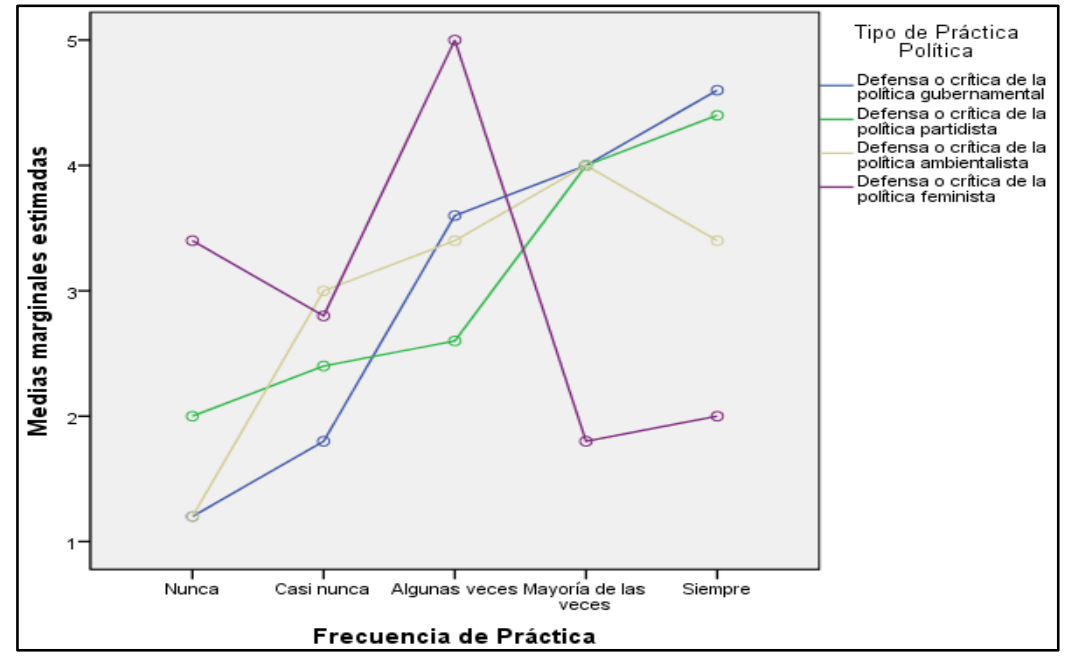

Fuente: Elaboración propia en base a las pruebas estadísticas en SPSS.

REVISTA ANUAL ACCIÓN Y REFLEXIÓN EDUCATIVA, N 45

enero, 2020

ISSN L 2664-3775 


\section{Prácticas de currículum oculto en la Dimensión Ideológica}

La Dimensión Ideológica, entendida como el conjunto de ideas sobre la realidad o prácticas de la sociedad respecto a lo económico, social, científico, político, cultural, moral, etc., está siempre presente en la humanidad y es difícil pensar que un profesor universitario pueda evitarla o eliminarla, utilizando únicamente la virtud teórica que ofrecen los libros de texto que utilizan para sus cursos. Si bien es importante función que cumplen los libros con respecto del conocimiento, también es innegable el papel que juega el profesor en la construcción de las ideas del alumnado.

La Dimensión Ideológica está estrechamente vinculada a la Dimensión Política, ya que es usual que los políticos basen su accionar en una ideología; ambos temas son a menudo controversiales. Los resultados se presentan a través de los gráficos lineales de medias. Se observan resultados similares, en los cuales, por un lado, las gráficas de los tipos de prácticas de la Dimensión Ideológica plantean una fuerte interacción, en tanto, que las frecuencias de las prácticas no se cruzan, pero aun así existe interacción significativa. Para el tipo de práctica, se evidencia que las medias marginales son muy cercanas, sin embargo, son más altas para las prácticas de "Defensa o crítica del capitalismo" y más bajas para la "Defensa o crítica del eclecticismo".

REVISTA ANUAL ACCIÓN Y REFLEXIÓN EDUCATIVA, N 45

enero, 2020

SSN L 2664-3775 
El Currículum oculto en la educación universitaria:

Un estudio de caso en la Universidad de Panamá

\section{Figura 7. Medias Marginales de la Dimensión Ideológica}

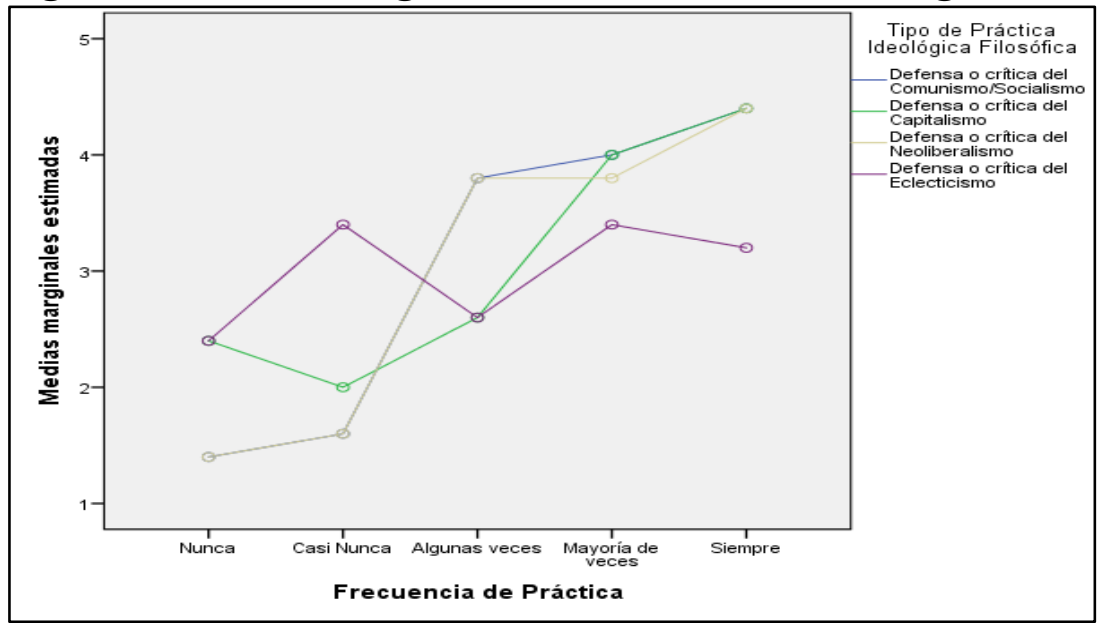

Fuente: Elaboración propia en base a las pruebas estadísticas en SPSS.

\section{Prácticas de Curriculum Oculto en la Dimensión de Valores}

El tema de valores es una práctica muy común no solo a nivel de la docencia superior sino también a nivel de la media y pre media. Los profesores con el fin de corregir conductas inapropiadas hacen llamados al orden apelando a los valores. Se presentan los gráficos lineales de perfil o gráficos de medias, donde se observan en las dos gráficas de medias marginales estimadas, que tanto para los tipos de prácticas de Currículum Oculto de la Dimensión Valores, como para las frecuencias de las prácticas existe interacción significativa, lo cual se corresponde con los resultados de las comparaciones múltiples. En el caso de las gráficas de las medias marginales por factor, podemos ver, que se confirma los valores más altos para la frecuencia de "Siempre" y "Algunas veces" y los valores más bajos para "Nunca" y "Casi nunca". Para el tipo de práctica, se evidencia que las medias marginales son muy cercanas, sin embargo, son más altas para las prácticas de "Discusión de valores humanos" y más bajas para la "Discusión de valores cívicos". 
Figura 8. Medias marginales de la Dimensión de Valores

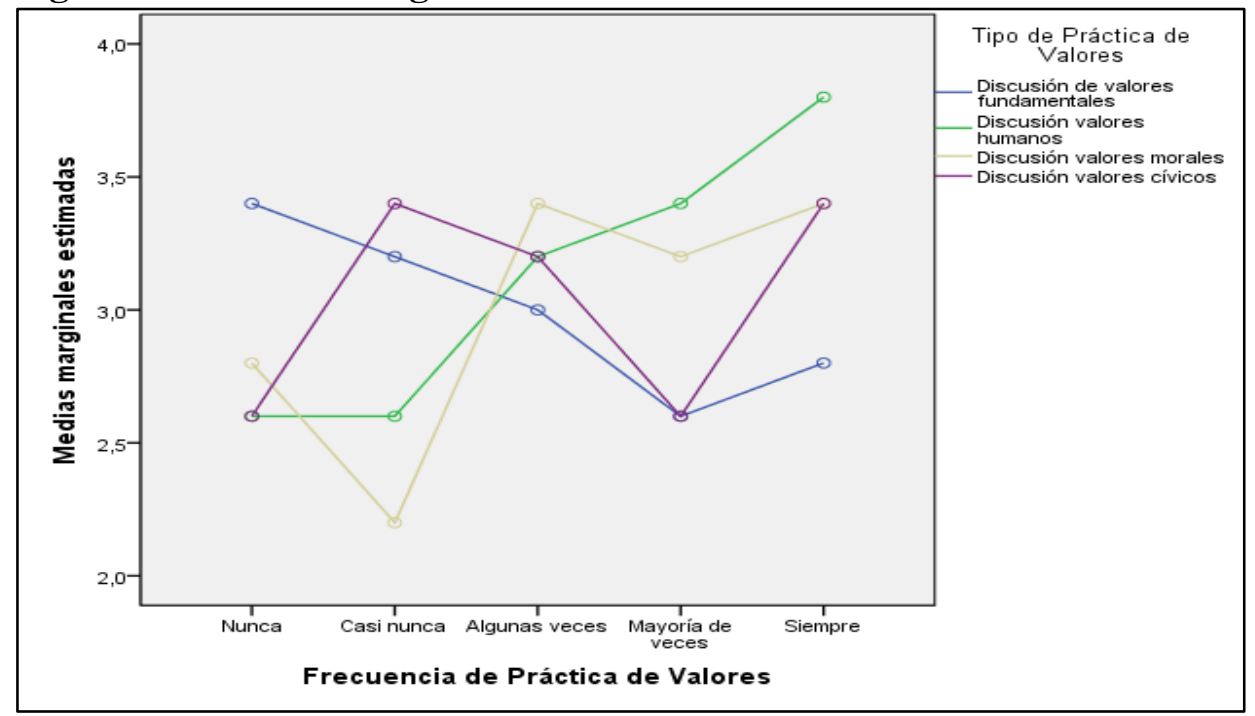

Fuente: Elaboración propia en base a las pruebas estadísticas en SPSS.

\section{Impacto de las prácticas de currículum oculto}

El segundo objetivo general de la investigación fue demostrar que las prácticas de Currículum Oculto de los profesores de la unidad académica seleccionada tienen un impacto positivo en el estudiante graduando. Para ello se desarrolló también la experimentación a través del ANOVA dos variables de pendientes: El Grado de Aceptación e impacto y el Grado de Influencia e impacto, con sus respectivos factores de interacción.

- Grado de aceptación e impacto: Para medir y evidenciar el Grado de Aceptación, se definió que los estudiantes deben seleccionar una entre las cuatro prácticas de Currículum Oculto y a su vez indicar los grados de aceptación que corresponden. Los gráficos de medias observan una fuerte interacción entre todas las dimensiones al igual que una concentración de las medias bajas en los grados de no aceptación y una concentración de medias altas en los grados de mayor aceptación. Con una sola excepción, la Dimensión Religiosa, siendo la única 
El Currículum oculto en la educación universitaria:

Un estudio de caso en la Universidad de Panamá

dimensión en la que los estudiantes manifestaron no estar de acuerdo y en total desacuerdo con sus prácticas por parte de los profesores.

Podemos ver, que para las dimensiones políticas, ideológicas y de valores son iguales; en tanto que para la dimensión religiosa la media está muy por debajo, confirmándose que las prácticas de esta dimensión no son del agrado de los estudiantes. Finalmente, a pesar de la discordancia de los estudiantes con respecto a las prácticas de la dimensión religiosa, en términos generales las medias marginales más altas, se agrupan en torno a que los estudiantes están "de acuerdo" y "muy de acuerdo" con las prácticas, lo cual nos lleva a concluir a través de la demostración gráfica que si existe interacción e influencia de la variable independiente o impacto del currículum oculto.

Figura 9. Medias marginales de Grado de Aceptación

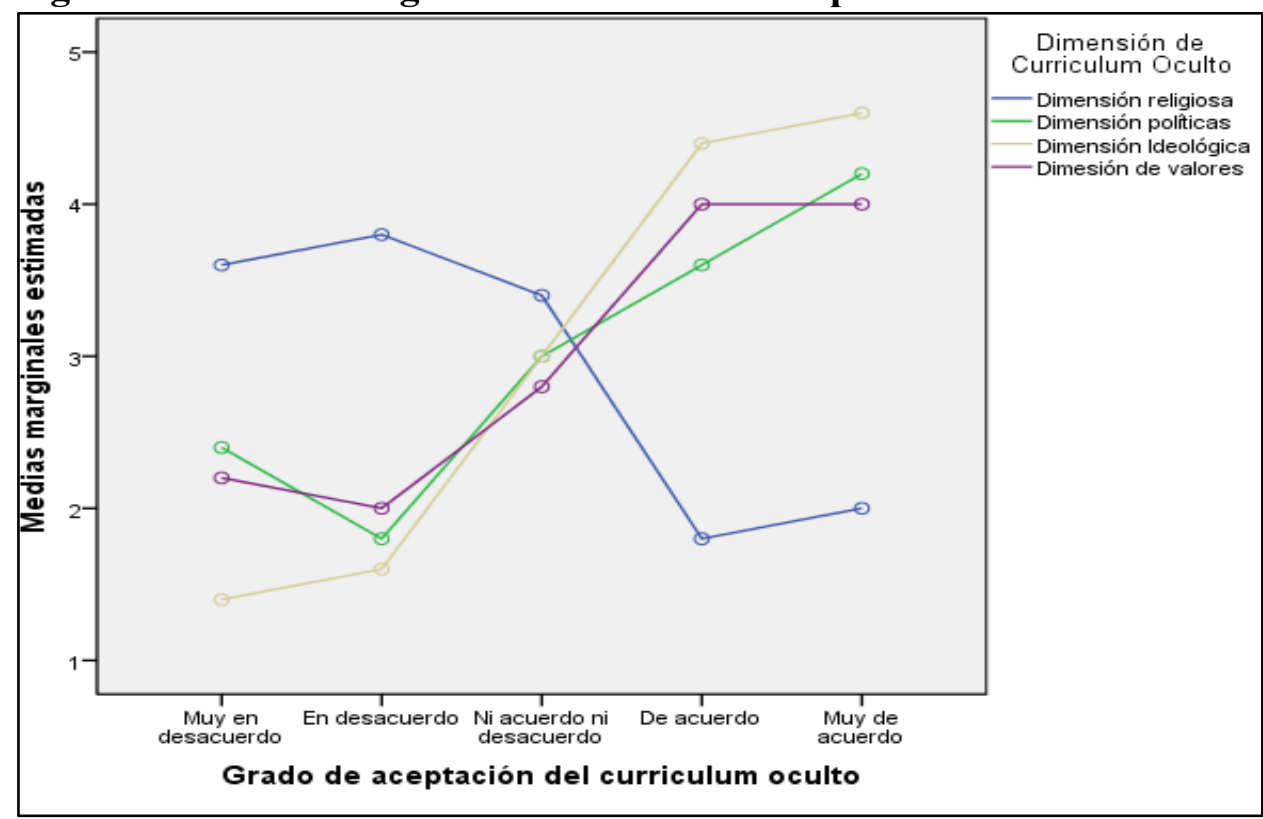

Fuente: Elaboración propia en base a las pruebas estadísticas en SPSS.

REVISTA ANUAL ACCIÓN Y REFLEXIÓN EDUCATIVA, N 45

enero, 2020

ISSN L 2664-3775 
- Grado de influencia e impacto: Con el interés de medir el grado de influencia e impacto de las prácticas del Currículum Oculto sobre el estudiante graduando, se les preguntó con respecto a los siguientes cuatro tipos de influencia: cambia su forma de pensar y actuar, afecta positiva o negativamente la formación profesional y son necesarias para el profesional universitario. Se presentan los gráficos, donde se observa una fuerte interacción entre todos los tipos de influencia, al igual que según los grados de influencia que también se cruzan, por tanto, la interacción es significativa. Las gráficas de las medias marginales podemos ver, que se para el tipo de influencia son iguales; con una sola excepción, la calificada como "Afecta negativamente la formación profesional" el cual obtuvo los valores promedios más bajos. Por su parte las medias marginales más altas se obtuvieron para "son necesarias para el profesional"'.

Figura 10. Medias marginales de Grado de Influencia

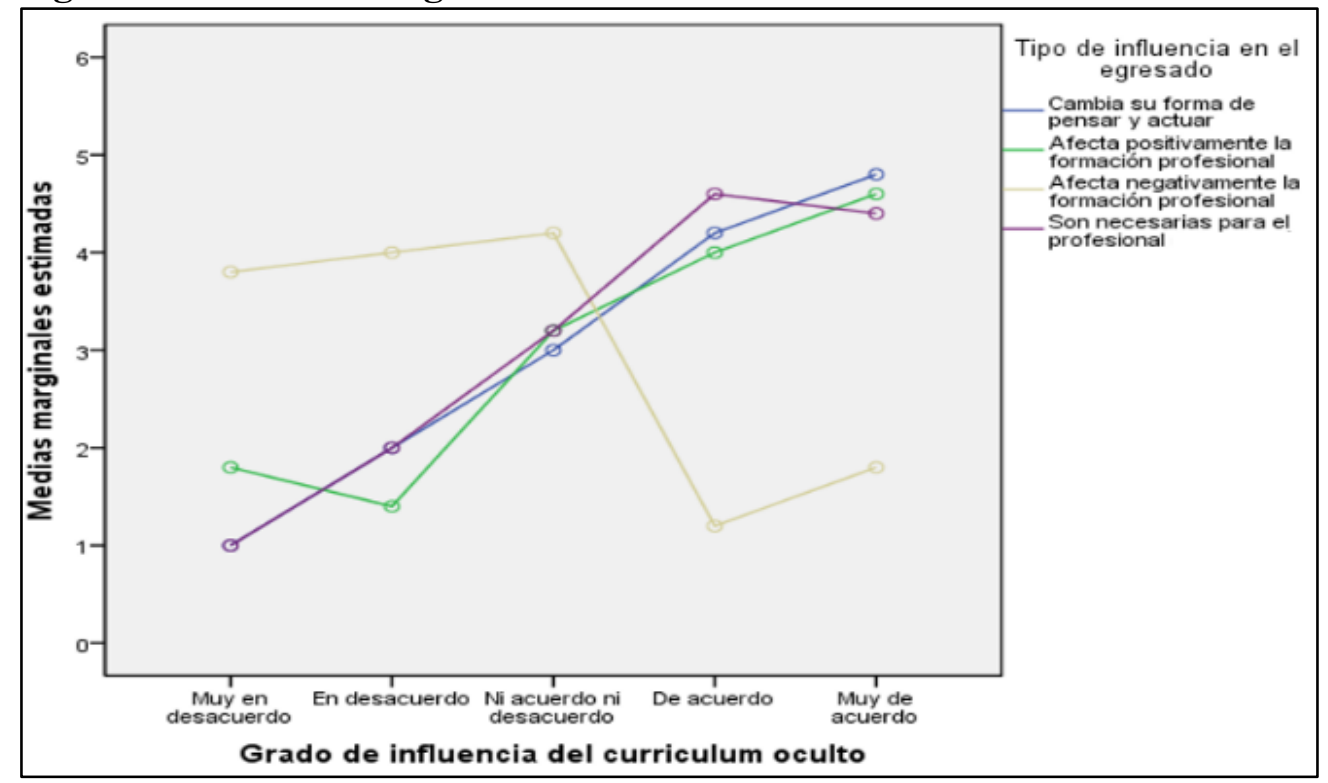

Fuente: Elaboración propia en base a las pruebas estadísticas en SPSS.

REVISTA ANUAL ACCIÓN Y REFLEXIÓN EDUCATIVA, N 45

enero, 2020

SSN L 2664-3775 
El Currículum oculto en la educación universitaria:

Un estudio de caso en la Universidad de Panamá

\section{Conclusiones}

Se confirma que el $85 \%$ de los encuestados(as) afirma que los profesores realizan prácticas de Currículum oculto, dichas prácticas son frecuentes en las unidades académicas seleccionadas, ya que los valores totales obtenidos a través del muestreo reflejan que los 73 estudiantes encuestados otorgaron calificaciones altas (4,571 puntos) en la frecuencia de "Siempre" a las prácticas de Currículum Oculto y calificaciones menores (2,316 puntos) en la frecuencia de "Nunca" de dichas prácticas. De igual forma, podemos concluir que las dimensiones Ideológica (1,198 puntos) y Religiosa (1,189 puntos) son las mayormente practicadas por los profesores y la Dimensión de Valores Éticos (1,009 puntos) es la menos practicada.

En cuanto al grado de aceptación de las prácticas de Currículum Oculto, los resultados indican que los estudiantes están "De acuerdo" y "Muy de acuerdo" con las prácticas, al igual que estos estudiantes están "Muy de acuerdo" en que las prácticas cambian su forma de pensar y actuar, en que las mismas son muy necesarias para el profesional y que afectan positivamente su formación. Sin embargo, las prácticas más aceptadas por los estudiantes fueron la dimensión de valores y la dimensión ideológica y la mayormente rechazada fue la práctica de la dimensión religiosa.

\section{Referencias}

Constitución Política de la República de Panamá. (2004). Reformada por los actos reformatorios de 1978, por el acto constitucional de 1983 y los actos legislativos 1 de 1983 y 2 de 1994. Gaceta Oficial No. 25176 del 15 de noviembre de 2004.

Eggleston, John. (1980). “Sociología del Currículum Escolar”. Primera edición Troquel, Buenos Aires, Argentina.

Giroux, Henry. (1992). "Teoría y Resistencia en Educación, una pedagogía para la oposición”. Buenos Aires, Argentina, Siglo XXI, editores S.A. $6^{\text {ta }}$, Edición; $1^{\mathrm{a}}$. Edición en español, título original: Theory and resistence in education pedagogy for the opposition. P. 332.

REVISTA ANUAL ACCIÓN Y REFLEXIÓN EDUCATIVA, N 45

enero, 2020

ISSN L 2664-3775 
Giroux, Henry. (1997). “Los profesores como intelectuales: Hacia una pedagogía crítica del aprendizaje”. Massachusetts, Estados Unidos Editorial Paidos. 7ª Edición.

Jackson, Philip W. (2001). "La Vida en las Aulas”, Sexta Edición (Reimpresión de 1968), Teachers College, Columbia University, Nueva York.

Lopes Da Silva, María José. (1996). “Un Aspecto de la Función Ideológica de la Escuela: o Currículo Oculto”, Boletín Técnico SENAC, Volumen 22, No.2 Rio de Janeiro, Brasil.

Torres Santomé, Jurjo. (1998). “El currículum oculto” Antomé Ediciones Morata, S.L., España.

Huerta, José, C. Osegueda; C. Mendoza, J. Castro. (1988). “El currículo en la práctica docente de la escuela de Odontología de la UAZ”, «Cuadernos de investigación», n 48, Zacatecas, México.

Universidad de Panamá. (2010). Boletín de Estadística Edición $N^{\circ} 76$, El Departamento de Estadística de la Dirección General de Planificación y Evaluación Universitaria, Primer Semestre del Año Académico 2010.

REVISTA ANUAL ACCIÓN Y REFLEXIÓN EDUCATIVA, N 45 enero, 2020 